\title{
Tuberculosis Intestinal Secundaria: Hallazgos Morfológicos en un Caso con Desenlace Fatal
}

\author{
Secondary Intestinal Tuberculosis: Morphological Findings in a Fatal Case \\ Oscar Tapia E.
}

TAPIA, E. O. Tuberculosis intestinal secundaria: hallazgos morfológicos en un caso con desenlace fatal. Int. J. Morphol., 30(1):347$353,2012$.

RESUMEN: La tuberculosis gastrointestinal (TB GI) representa el 3-5\% de todos los casos de TB extrapulmonar, reportándose que hasta el 20\% de los sujetos con TB pulmonar presentan manifestaciones extra-pulmonares que incluyen enfermedades intra-abdominales, sin embargo, se desconoce la real incidencia del compromiso intestinal. La región ileocecal representa el sitio anatómico más frecuentemente afectado (85-90\%), siendo actualmente la colonoscopía la herramienta diagnóstica de mayor utilidad. Se presentan los hallazgos morfológicos del examen post-mortem de una paciente en estudio por baja de peso y masa en región cecal. La necropsia realizada en la Unidad de Anatomía Patológica del Hospital Hernán Henríquez Aravena de Temuco concluyó una TB pulmonar bilateral con compromiso secundario intestinal y linfonodal.

PALABRAS CLAVE: Tuberculosis; Gastrointestinal; Tuberculosis intestinal.

\section{INTRODUCCIÓN}

La tuberculosis (TB) es una antigua enfermedad, que continúa hoy en día representando un problema en salud pública en el mundo, dado los niveles de pobreza, resistencia a las drogas y la epidemia del virus de la inmunodeficiencia humana (VIH). La Organización Mundial de la Salud (OMS), estima que un tercio de la población del mundo está infectado por Mycobacterium tuberculosis, representando esta causa el mayor número de muertes de origen infeccioso. Su incidencia es de aproximadamente 8.8 millones, dando cuenta de alrededor de $1,5 \mathrm{mi}-$ llones de muertes anuales (Erhabor et al., 2010; Guth \& Kim, 1991; Horvath \& Whelan, 1998; WHO, 2008).

La TB gastrointestinal representa el 3-5\% de todos los casos de TB extrapulmonar, reportándose que hasta el $20 \%$ de los sujetos con TB pulmonar presentan manifestaciones extra-pulmonares que incluyen enfermedades intraabdominales, sin embargo, se desconoce su real incidencia (Hamer \& Gorbach, 1998; Marshall, 1993; Rasheed et al., 2007).

La patogenia de la TB gastrointestinal se produce por la ingesta de alimentos o esputos contaminados, por diseminación hematógena de una tuberculosis pulmonar activa o miliar o por afectación de otros órganos contiguos (Horvath \& Whelan). Si bien puede afectarse cualquier porción del tubo digestivo, la región ileocecal representa el sitio anatómico más frecuentemente afectado (85-90\%) seguido de colon ascendente (35\%) transverso (16\%) y recto-sigmoides (13\%) (Chong \& Lim, 2009).

Se presentan los hallazgos morfológicos del examen post-mortem de una paciente de 46 años en estudio por baja de peso y masa en región cecal. La necropsia realizada en la Unidad de Anatomía Patológica del Hospital Hernán Henríquez Aravena de Temuco concluyó una TB pulmonar bilateral con compromiso secundario intestinal y linfonodal regional.

\section{REPORTE DEL CASO}

Mediante técnica de Virchow modificada se examinó cadáver de adulto de sexo femenino, de constitución ectomórfica de Sheldon. 


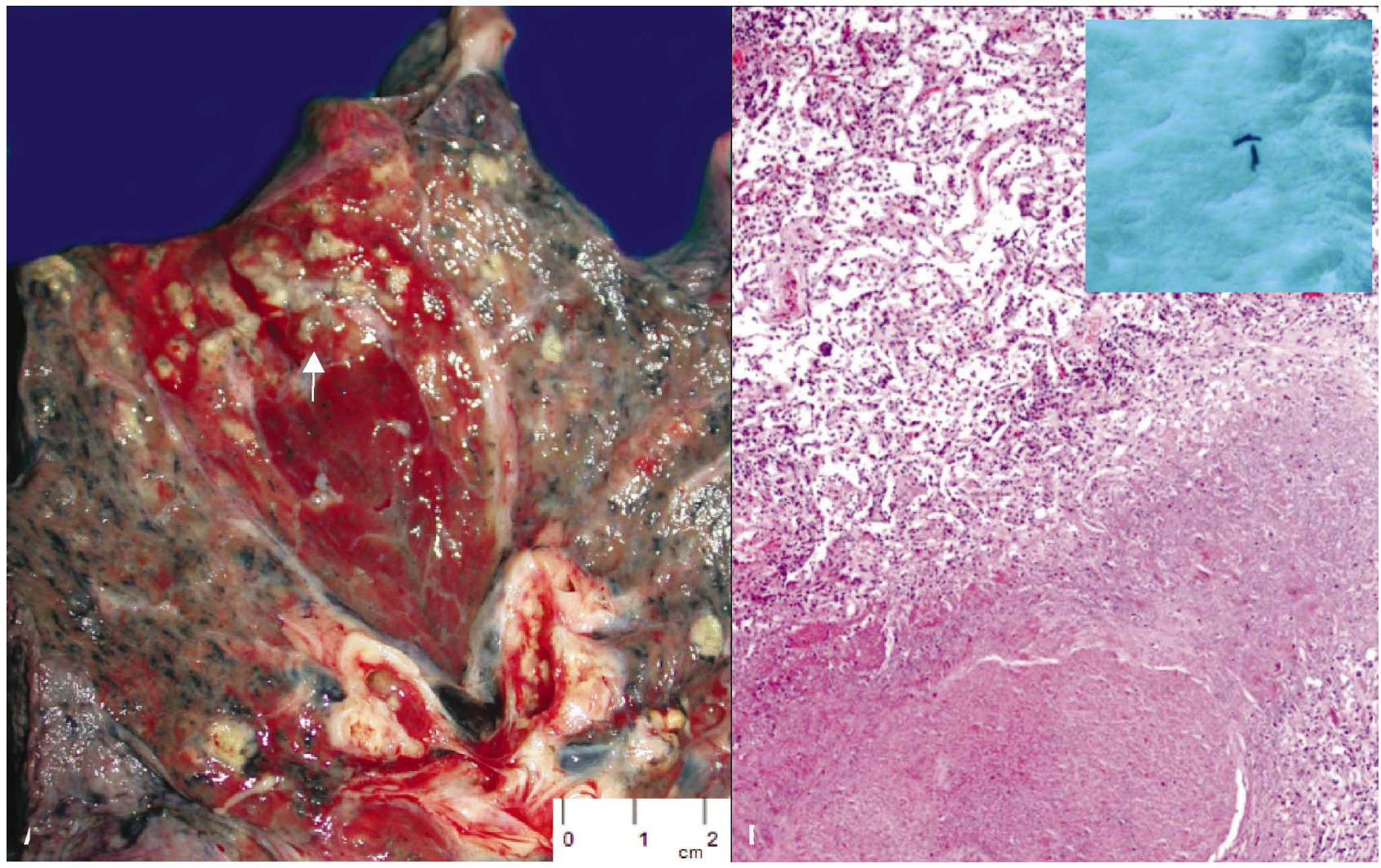

Fig. 1. Pulmón. A. Corte longitudinal, ápex pulmón izquierdo. En la superficie de corte se reconocen áreas nodulares pardo amarillentas (flecha) de aspecto caseoso. B. Se aprecia parénquima pulmonar con proceso inflamatorio granulomatoso con focos necróticos caseosos y presencia de bacilos ácido alcohol resistentes con la tinción de Ziehl Neelsen (recuadro) (40x, H-E).

En ambas cavidades pleurales se observó líquido amarillento, $680 \mathrm{ml}$ en hemitórax derecho y $780 \mathrm{ml}$ en hemitórax izquierdo. El pulmón izquierdo y derecho pesaron $400 \mathrm{~g}$ y $847 \mathrm{~g}$ respectivamente. Bronquios lobares y ramas de la arteria pulmonar sin lesiones. Superficie externa de ambos pulmones parda violácea con áreas de antracosis maculo-reticular y zonas induradas en región apical y media del lóbulo superior izquierdo y lóbulo medio de pulmón derecho. Al corte, la superficie pulmonar rojo violácea; en zona apical izquierda área pardo grisácea con material de aspecto caseoso y formación de pequeños nódulos múltiples (Fig. 1A). Pulmón derecho de consistencia aumentada, superficie rojo violácea con zonas pardo amarillentas nodulares reblandecidas y de aspecto caseoso. El examen microscópico mostró pulmones de estructura conservada, con hiperemia capilar septal y focos de hemorragia intersticial e intra-alveolar con presencia de infiltrado inflamatorio polimorfonuclear en partes con disrupción de tabiques alveolares y bronquiolares. Se observaron múltiples focos redondeados de necrosis caseosa con escasa formación de empalizada epiteloídea y células gigantes de Langhans demostrando la tinción de Zeihl Neelsen la presencia bacilos ácido alcohol resistentes (Fig. 1B). En región carinal y peritraqueal se observaron linfonodos aumentados de tamaño y formando conglomerados con presencia al examen microscópico de focos de necrosis caseosa con células gigantes de Langhans y bacilos ácido alcohol resistentes en la tinción de Zeihl Neelsen (Figura 2A).

En cavidad abdominal presencia de $300 \mathrm{ml}$ de líquido amarillento. El mesenterio presentó pequeñas formaciones nodulares y opacas, blanquecinas junto con conglomerados de adenopatías mesentéricas con áreas de necrosis caseosa y formación de una empalizada por células epiteloídeas con ausencia de células gigantes de Langhans y presencia de escasos bacilos ácido alcohol resistentes con tinción de Ziehl Neelsen (Figura 2B). Los $120 \mathrm{~cm}$ distales de intestino delgado presentaron múltiples zonas granulares en partes nodulares pardo blanquecinas y opacas de hasta $0,4 \mathrm{~cm}$ con depósito de material fibrinoídeo y pared intestinal engrosada en par- 


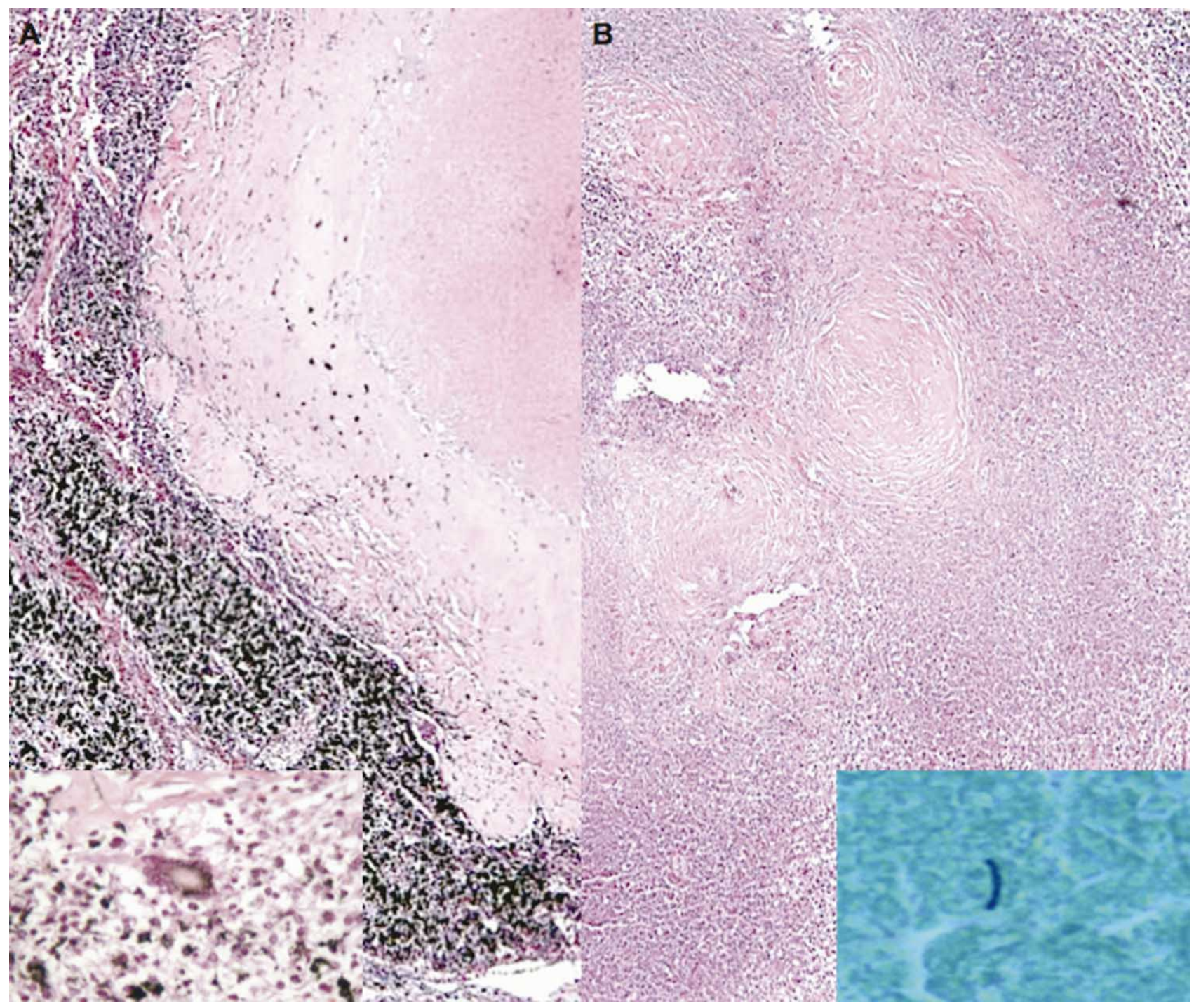

Fig. 2. Linfonodos. A. Linfonodo peri-traqueal y B. Linfonodo mesentérico (40x, H-E). Linfonodos de arquitectura distorsionada con proceso inflamatorio productivo-caseoso con presencia de células gigantes de Langhans (recuadro izquierdo) y bacilos ácido alcohol resistentes con tinción de Ziehl Neelsen (recuadro derecho).

tes retraída (3A). La mucosa intestinal presentó múltiples áreas ulceradas de bordes solevantados e irregulares de aspecto trabecular formando anillos estenóticos que alternaba con áreas de mucosa conservada. El intestino grueso presentó en la región ileocecal área irregular nodular y ulcerada de aproximadamente 6 x $6 \mathrm{~cm}$ con engrosamiento parietal y compromiso del tejido adiposo peri-cecal, el cual se encontraba indurado y con nódulos blanquecino-amarillentos de aspecto caseoso (Figura 3B-D). En la microscopía se observó a nivel de intestino delgado y ciego múltiples erosiones y úlceras con fondo necrótico, infiltrado inflamatorio redondo celular y formación de tejido granulatorio, en partes con presencia de focos necróticos de tipo caseoso con formación de empalizada epiteloídea sin presencia de células gigantes de tipo Langhans. La tinción de Ziehl Neelsen demostró bacilos ácido alcohol resistentes (Figura 3E-F). Del mismo modo, el estudio de PCR para detección de M. tuberculosis resultó positivo (Fig. 4).

El examen de autopsia concluyó una tuberculosis pulmonar acinar bilateral activa con linfoadenitis mediastínica tuberculosa predominantemente exudativa y compromiso tuberculoso intestinal secundario (tipo mixto, ulcerativa-hipertrofica) 

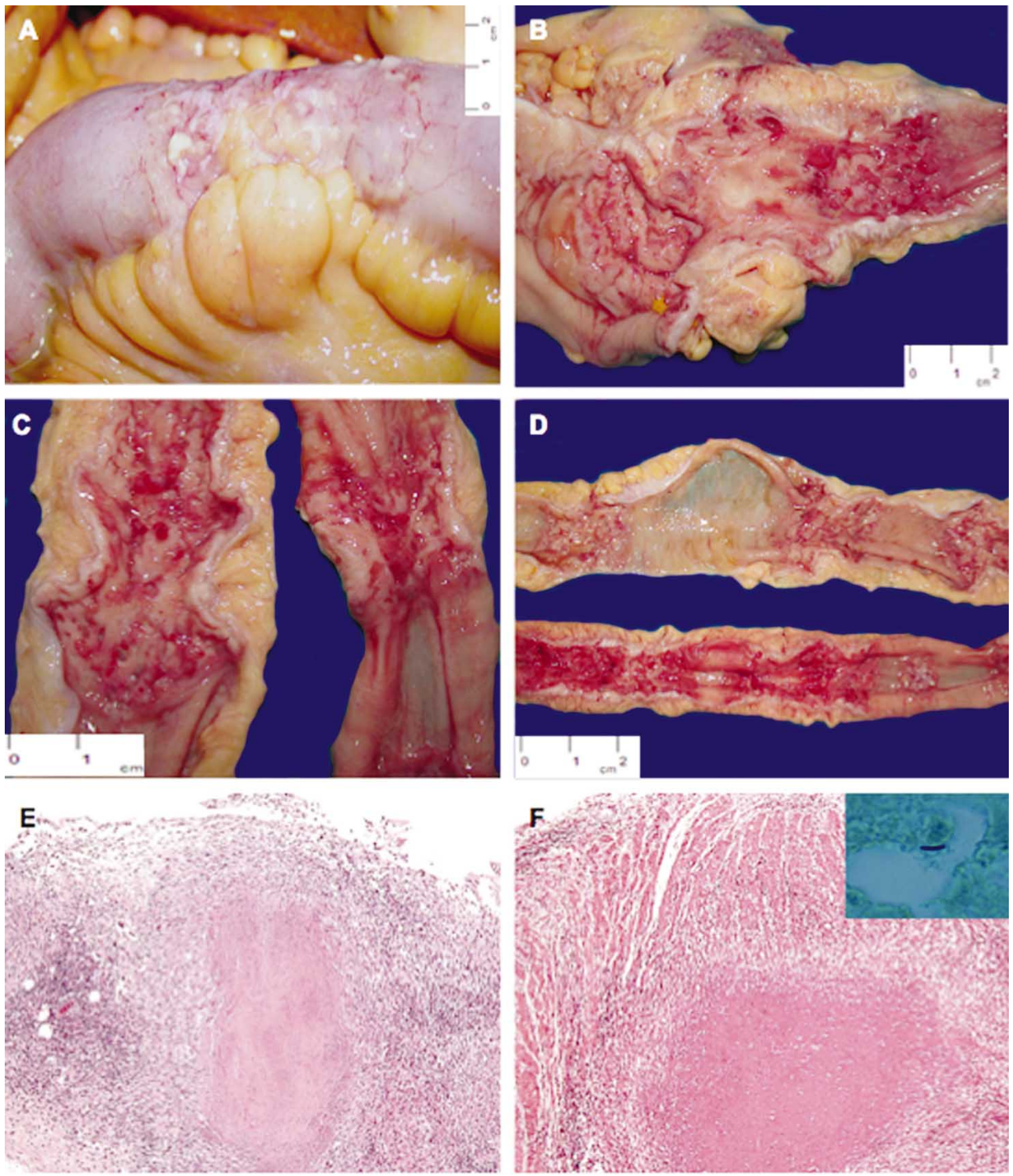

Fig. 3. Intestino. A. Ileon. Serosa de intestino delgado con vasos sanguíneos suberosos congestivos. Depósito de material fibrinoídeo y pequeños nódulos amarillentos de consistencia blanda. B. Región ileo-cecal. Mucosa de arquitectura distorsionada por múltiples zonas ulceradas con focos de hemorragia y disminución del lumen que comprometen ileon distal y ciego. C-D. Yeyuno-ileon. Múltiples úlceras circunferenciales parcialmente estenóticas, algunas de ellas con engrosamiento parietal y formación de trabéculas. E-F. Pared de intestino delgado acentuadamente distorsionada, con erosión superficial y formación de úlceras con proceso inflamatorio productivo-caseoso y presencia de bacilos ácido alcohol resistentes con tinción de Ziehl Neelsen (F recuadro) (40x, H-E). 


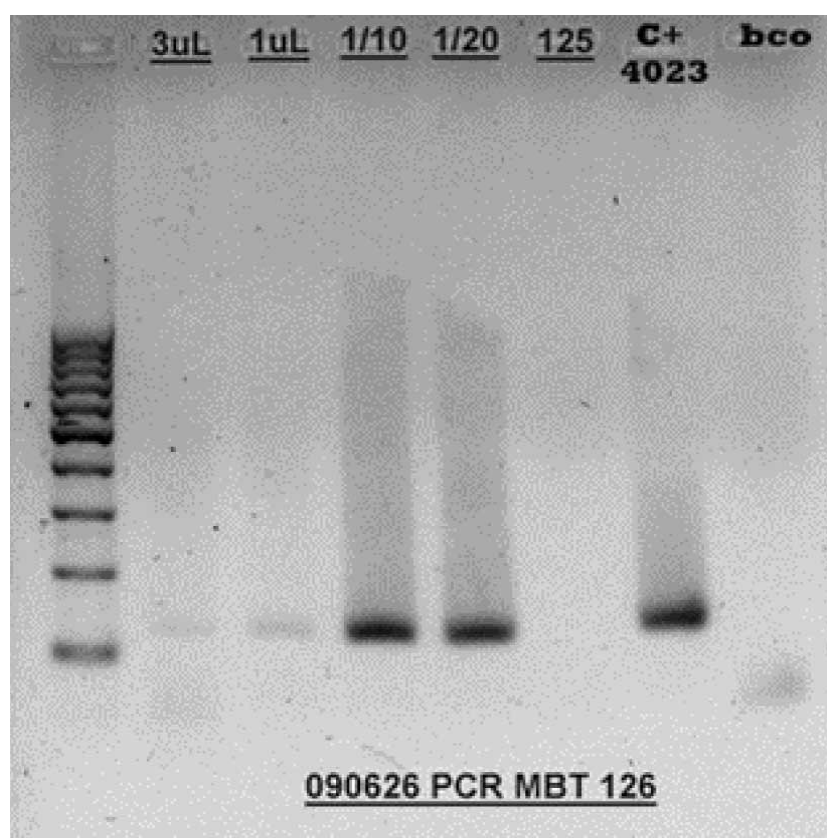

Fig. 4. Intestino delgado. Reacción en cadena de la polimerasa (PCR) con amplificación del ADN de M. tuberculosis.

\section{DISCUSIÓN}

La TB gastrointestinal representa el 3-5\% de todos los casos de localización extrapulmonar, pudiendo afectar cualquier porción del tubo digestivo; estando relacionada la prevalencia del compromiso gastrointestinal con la gravedad o intensidad de la enfermedad pulmonar, de esta forma la afección GI en sujetos con TB pulmonar leve ocurre aproximadamente en el $1 \%$, mientras que en pacientes con enfermedad extensa hasta un $25 \%$ presentara compromiso intestinal (Chong \& Lim; Hamer \& Gorbach; Marshall). De este modo, el resurgimiento de la TB en los últimos años, explicado por la pandemia del VIH, mayor uso de medicamentos inmunosupresores y envejecimiento población, ha producido también un aumento en la incidencia de TB intestinal, desconociéndose, sin embargo, la real frecuencia de esta enfermedad (Erhabor et al.; Guth \& Kim; Horvath $\&$ Whelan; WHO).

La patogenia de la tuberculosis intestinal se explica por cuatro mecanismos: 1) diseminación hematógena de tuberculosis pulmonar activa o miliar, 2) por deglución de esputo en pacientes con tuberculosis pulmonar activa, 3) ingestión de leche o alimentos contaminados y 4) diseminación contigua de órganos adyacentes (Horvath \& Whelan). Nuestra paciente presentaba una TB pulmonar activa, explicándose el compromiso intestinal secundario a la deglución de esputo contaminado.
Morfológicamente y dependiendo de la respuesta inmune del huésped, la TB GI se puede presentar en tres formas: ulcerosa (60\%), hipertrófica (10\%) y mixta o ulcerohipertrófica (30\%); la forma ulcerosa se produce más frecuentemente en pacientes inmunodeprimidos mientras que la hipertrófica en inmunocompetentes. Las úlceras son circunferenciales, con su eje mayor perpendicular al eje mayor intestinal, razón por la cual tienden a formar anillos fibrosos estenóticos cuando curan. La forma hipertrófica consiste en un engrosamiento fibroso y cicatricial de la pared intestinal, dándole una apariencia rígida que puede semejar una neoplasia maligna. La forma mixta en tanto combina elementos de ambas (Akgun, 2005; Chong \& Lim; Hamer \& Gorbach; Leung et al., 2006). El segmento intestinal más frecuentemente afectado corresponde a la región ileocecal (80-95\%), explicado esto por la abundancia de tejido linfoide, $\mathrm{pH}$ neutro y mínima actividad digestiva de esta zona, causando un mayor tiempo de contacto entre el bacilo y la mucosa intestinal (Sharma \& Bhatia, 2004). Otros sitios comprometidos son colon ascendente (35\%) transverso (16\%) y recto-sigmoides (13\%), mientras que duodeno y ano son rara vez afectados (Akgun; Chong \& Lim; Leung et $a l$.). En el caso reportado se observó compromiso de la región ileo-cecal y gran parte del ileon y yeyuno, correspondiendo estas lesiones a la forma mixta.

El diagnóstico de TB intestinal en ocasiones resulta difícil, pues los hallazgos clínicos y de laboratorio son inespecíficos, y tan solo el $15-25 \%$ de los casos presenta compromiso pulmonar concomitante (Faylona \& Chung, 1993; Horvath \& Whelan). La confirmación diagnóstica requiere demostrar la presencia de $M$. tuberculosis a partir del tejido afectado o evidencia de TB en otro sitio con presencia de granulomas caseificantes en el intestino, siendo la colonoscopía con obtención de muestras para estudio histopatológico la herramienta diagnóstica más útil (Bhargava et al., 1985; Kim et al., 1998; Shah et al., 1992; Singh et al., 1996). Los hallazgos histológicos típicos consideran la presencia de granulomas caseosos con células gigantes de Langhans, sin embargo, su presencia en muestras endoscópicas es muy variable (0-44\%), por lo que su ausencia ante la sospecha clínica no debe descartarla, pues se localizan en las capas profundas de la pared intestinal, hecho que explica que en piezas quirúrgicas de pacientes operados por complicaciones (obstrucción intestinal, hemorragia o perforación), se identifiquen con mayor frecuencia. Junto con esto, la demostración de bacilos ácido alcohol resistentes con la tinción de Ziehl Neelsen confirmarían el diagnóstico, resultando positiva entre $35-60 \%$ de los casos. Ahora bien, en casos de ausencia de hallazgos histológicos típicos o no demostrar la presencia de bacilos ácido alcohol resistentes en la tinción de Ziehl Neelsen y de persistir la sospecha clínica, la amplificación del ADN de M. tuberculosis 
mediante la reacción en cadena de la polimerasa (PCR) sería de utilidad en estos casos (Bhargava et al.; Chatzicostas et al., 2002; Kim et al.; Misra et al., 1999; Shah et al.; Singh et al.). En este caso evidenciamos la presencia de granulomas caseosos con escasas células gigantes de Langhans, demostrándose con Ziehl Neelsen bacilos ácido alcohol resistentes confirmado esto mediante la amplificación del ADN de M. tuberculosis mediante PCR

La mortalidad de la TB intestinal sin mediar tratamiento farmacológico es de 20-50\%, disminuyendo a 2-21\% con tratamiento anti-tuberculoso oportuno. La obstrucción intestinal es la complicación más frecuente (15-60\%), otras complicaciones son fístulas (25\%) y perforación en el 15\% de los casos, con una tasa de mortalidad de hasta $40 \%$ para estos pacientes (Akgun; Bhansali et al., 1968; Bhansali,
1977; Faylona \& Chung; Lingenfelser et al., 1993; Ruiz Gómez et al,. 2006; Serf et al., 2001; Sharma \& Bhatia).

En resumen, el aumento en la incidencia de TB pulmonar y secundariamente del compromiso intestinal, hacen que hoy el médico clínico deba tener un alto índice de sospecha frente a pacientes con TB pulmonar y sintomatología abdominal, siendo el estudio endoscópico con toma de muestras para estudio anatomo-patológico de las lesiones sospechosas de utilidad diagnóstica, teniendo siempre presente los posibles falsos negativos. Del mismo modo el médico clínico y anatomopatólogo deben estar familiarizado con la morfología, cambios histo-patológicos y complicaciones asociadas a esta enfermedad, de manera de efectuar un diagnóstico oportuno y adecuado que permitan la instauración precoz de terapias sistémicas antituberculosas y/o quirúrgicas.

TAPIA, E. O. Secondary intestinal tuberculosis: morphological findings in a fatal case. Int. J. Morphol., 30(1):347-353, 2012.

SUMMARY: Gastrointestinal tuberculosis (TB GI) represents 3-5\% of all cases of extrapulmonary TB, studies report that up to $20 \%$ of patients with pulmonary TB have extra-pulmonary manifestations. However, the real incidence of intestinal involvement is unknown. The ileocecal region is the anatomical site most frequently affected (85-90\%), and colonoscopy is currently the most useful diagnostic tool. Post-mortem morphological elements of the examination of a patient with weight loss and cecal mass are presented. The necropsy performed at the Department of Pathology of the Hospital Hernán Henríquez of Temuco concluded a bilateral pulmonary TB with intestinal and lymph node involvement.

KEY WORDS: Tuberculosis; Gastrointestinal; Intestinal tuberculosis.

\section{REFERENCIAS BIBLIOGRÁFICAS}

Akgun, Y. Intestinal and peritoneal tuberculosis: changing trends over 10 years and a review of 80 patients. Can. J. Surg., 48(2):131-6, 2005

Bhansali, S. K.; Desai, A. N. \& Dhaboowala, C. B. Tuberculous perforation of the small intestine. A clinical analysis of $19 \mathrm{ca}-$ ses. J. Assoc. Physicians India, 16(6):351-5, 1968.

Bhansali, S. K. Abdominal tuberculosis. Experiences with 300 cases. Am. J. Gastroenterol., 67(4):324-37, 1977.

Bhargava, D. K.; Tandon, H. D.; Chawla, T. C.; Shriniwas; Tandon, B. N. \& Kapur, B. M. Diagnosis of ileocecal and colonic tuberculosis by colonoscopy. Gastrointest. Endosc., 31(2):68-70, 1985.

Chatzicostas, C.; Koutroubakis, I. E.; Tzardi, M.; Roussomoustakaki, M.; Prassopoulos, P. \& Kouroumalis, E. A. Colonic tuberculosis mimicking Crohn's disease: case report. BMC Gastroenterol., 2:10, 2002.

Chong, V. H. \& Lim, K. S. Gastrointestinal tuberculosis. Singapore Med. J., 50(6):638-46, 2009.

Erhabor, O.; Jeremiah, Z. A.; Adias, T. C. \& Okere, C. The prevalence of human immunodeficiency virus infection among
TB patients in Port Harcourt Nigeria. HIV AIDS, 2:1-5, 2010.

Faylona, J. M. \& Chung, S. C. Abdominal tuberculosis revisited. Ann. Coll. Surg., 3:65-70, 1993.

Guth, A. A. \& Kim, U. The reappearance of abdominal tuberculosis. Surg. Gynecol. Obstet., 172(6):432-6, 1991.

Hamer, D. H. \& Gorbach, S. L. Tuberculosis of the intestinal tract. In: Felman, M.; Scharschmidt, B. F. \& Sleisenger, M. H. (Eds.). Sleisenger and Fordtrans's Gastrointestinal and Liver Disease. Pathophysiology/Diagnosis/Management. Vol 2. 6th ed. Philadelphia, WB Saunders, 1998. pp.1622-4.

Horvath, K. D. \& Whelan, R. L. Intestinal tuberculosis: return of an old disease. Am. J. Gastroenterol., 93(5):692-6, 1998.

Kim, K. M.; Lee, A.; Choi, K. Y.; Lee, K. Y. \& Kwak, J. J. Intestinal tuberculosis: clinicopathologic analysis and diagnosis by endoscopic biopsy. Am. J. Gastroenterol., 93(4):606-9, 1998.

Leung, V. K.; Law, S. T.; Lam, C. W.; Luk, I. S.; Chau, T. N.; Loke, T. K.; Chan, W. H. \& Lam, S. H. Intestinal tuberculosis in a regional hospital in Hong Kong: a 10-year experience. Hong Kong Med. J., 12(4):264-71, 2006. 
Lingenfelser, T.; Zak, J.; Marks, I. N.; Steyn, E.; Halkett, J. \& Price, S. K. Abdominal tuberculosis: still a potentially lethal disease. Am. J. Gastroenterol., 88(5):744-50, 1993.

Marshall, J. B. Tuberculosis of the gastrointestinal tract and peritoneum. Am. J. Gastroenterol., 88(7):989-99, 1993.

Misra, S. P.; Misra, V.; Dwivedi, M. \& Gupta, S. C. Colonic tuberculosis: clinical features, endoscopic appearance and management. J. Gastroenterol. Hepatol., 14(7):723-9, 1999.

Rasheed, S.; Zinicola, R.; Watson, D.; Bajwa, A. \& McDonald, P. J. Intra-abdominal and gastrointestinal tuberculosis. Colorectal Dis., 9(9):773-83, 2007.

Ruiz Gómez, J. L.; García Díaz, R. A.; Rodríguez San Juan, J. C.; García Palomo, D. \& Gómez Fleitas, M. Perforated intestinal tuberculosis in an immunocompetent patient. Rev. Esp. Enferm. Dig., 98(10):793-4, 2006.

Sefr, R.; Rotterová, P. \& Konecnł, J. Perforation peritonitis in primary intestinal tuberculosis. Dig. Surg., 18(6):475-9, 2001.

Shah, S.; Thomas ,V.; Mathan, M.; Chacko, A.; Chandy, G.; Ramakrishna, B. S. \& Rolston, D. D. Colonoscopic study of 50 patients with colonic tuberculosis. Gut, 33(3):347-51, 1992.

Sharma, M. P. \& Bhatia, V. Abdominal tuberculosis. Indian J. Med. Res., 120(4):305-15, 2004

Singh, V.; Kumar, P.; Kamal, J.; Prakash, V.; Vaiphei, K. \& Singh, K. Clinicocolonoscopic profile of colonic tuberculosis. Am. J. Gastroenterol., 91(3):565-8, 1996.

World Health Organisation (WHO). Global Tuberculosis Control: Surveillance, Planning. Financing: WHO report 2008. Geneva, World Health Organization, 2008.

\author{
Dirección para correspondencia: \\ Dr. Oscar Tapia E. \\ Departamento de Anatomía Patológica \\ Facultad de Medicina, Universidad de La Frontera \\ Manuel Montt 112. Código Postal 478-1176. \\ Fono: 045-296530 \\ Temuco, CHILE \\ Email: otescalona@gmail.com
}

Recibido : 21-03-2011

Aceptado: 29-11-2011 